Revista de Psicología de la PUCP. Vol. XV, 2, 1997.

\title{
EL MEJORAMIENTO DE LA CAPACIDAD PARA APRENDER A TRAVÉS DE LA LECTURA
}

\author{
Armando Morles, Ph.D. ${ }^{1}$ \\ Universidad Pedagógica Experimental Libertador
}

Ll presente estudio discute la importancia de la instrucción en la capacidad para aprender el contenido de textos escritos. Al inicio se define el aprendizaje a través de la lectura para luego continuar con la identificación de los factores que condicionan este aprendizaje. Luego se suministra lineamientos para mejorar esa capacidad. Finalmente se aportan ideas acerca de cómo conducir el entrenamiento de los estudiantes, incluyendo las modalidades y su ubicación dentro del contexto educativo.

Palabras claves: lectura, aprendizaje, textos.

The improvement of learning ability by means of reading

This study discusses the importance of instruction in the ability to learn from texts. First learning through reading is defined, followed by the identificaction of the factors that determine this learning. It provides guidelines for the improvement of such an ability. Finally, it gives ideas about ways of conducting the student training, including exercising modalities and the program placement in the educational context.

Keywords: reading, learning, texts.

1. Psicólogo educativo egresado de la Universidad Central de Venezuela. Con estudios de maestría, especialización y doctorado de la Universidad de Chicago (US $\Lambda$ ). Docente-Investigador en las áreas de la lectura, los materiales escritos y el desarrollo cognoscitivo. $\Lambda$ sesor de Ministerios de Viducación y consultor de organismos internacionales en los países latinoamericanos. Apartado Postal 2939 Caracas 1010, Venezuela. Teléfonos: 582-923929, 582-837822, 582-837922. Fax: (582) $817-227$ y (582) 662-7455. 

Es una realidad aceptada en el presente que la capacidad para aprender el contenido de los textos escritos puede ser mejorada significativamente. Es obvio que para lograr este mejoramiento se requiere de una tecnología de instrucción que precise sobre cuáles aspectos se debe actuar y cómo se debe conducir esa actuación. Hay muchas maneras de atender a esta problemática y el presente trabajo suministra una de ellas. Esta ha demostrado ser apropiada para el diseño de acciones dentro del sistema educativo regular y con los docentes ordinarios que laboran en él.

\section{¿Qué es el aprendizaje a través de la lectura?}

La acción educativa se apoya cada día con mayor fuerza en el aprendizaje obtenido a través de la lectura; es decir, en el aprendizaje de la información transmitida en materiales escritos. Esta situación es consecuencia del marcado incremento de la demanda por educación, en todos los niveles y áreas de los sistemas educativos, acompañada de la creciente escasez de recursos para atender apropiadamente a esa demanda. Tal hecho ha conducido a quienes planifican y dirigen la educación a recurrir a tecnologías que reduzcan los costos de la actuación educativa y aumenten la cobertura de esa atención. Un resultado de esta manera de actuar lo constituye el incremento en el uso de materiales escritos como una estrategia para satisfacer la demanda mencionada.

Por tal razón, la educación está dependiendo cada día más de la efectividad de los materiales escritos, de la capacidad para leer de los estudiantes y, más específico monto aún, de la capacidad de éstos para aprender a través de la lectura.

La efectividad de los materiales escritos como medio de aprendizaje es crucial, por lo cual a este aspecto se le ha venido dedicando marcada 
atención en los últimos tiempos (ver Morles, 1986a, 1986b y 1991). Sin embargo, es mucho lo que aún se necesita hacer para garantizar esa efectividad.

Con respecto a la capacidad para aprender conviene tener presente que aunque en lo relacionado con el aprendizaje en general existe una enorme masa de investigación, en lo que se refiere al aprendizaje a través de la lectura la historia es menos pródiga y menos variada. Este tipo de aprendizaje presenta características muy particulares, por lo que su consideración y tratamiento requieren de enfoques igualmente muy particulares.

En el presente, la psicología cognoscitiva ha dado aportes significativos a la búsqueda de maneras efectivas para atender este tipo de aprendizaje. En tal sentido esta disciplina comenzó por caracterizarlo como un proceso que incluye un conjunto de operaciones mentales (estrategias) que ejecuta quien intenta aprender, con el propósito de procesar la información contenida en los materiales escritos para, de esa manera, comprenderla, incorporarla a su memoria y estar en condiciones de evocarla cuando las circunstancias así lo requieran.

El procesamiento señalado comprende cinco subprocesos o etapas claramente identificables, aun cuando en la manera como se suceden pudiera existir marcada superposición entre todos ellos. Estos subprocesos o etapas son: la percepción de lo escrito, la decodificación o desciframiento de lo escrito, la obtención del significado de la información contenido, en lo escrito (comprensión), el almacenamiento de esa información (retención) y la evocación de la misma, motivada por variados propósitos.

Los tres primeros subprocesos constituyen lo que comúnmente se denomina lectura; mientras que esta lectura, complementada por la retención y la evocación constituye el aprendizaje de la información escrita.

El desarrollo de las habilidades relacionadas con la percepción de lo escrito y su decodificación es suficientemente atendida en los primeros años de la educación primaria. Esto se hace durante la enseñanza de lo 
que tradicionalmente se conoce como la mecánica de la lectura y, en general, los estudiantes presentan buen dominio de estas habilidades. En cambio, el desarrollo de las habilidades para la comprensión de lectura, la retención y la evocación son comúnmente descuidadas en las escuelas, lo cual es inferible a partir de la consideración del poco dominio que, generalmente, presentan los estudiantes en estas habilidades. Por esta razón el presente trabajo está referido fundamentalmente al desarrollo de este tipo de habilidades.

\section{Factores que condicionan el aprendizaje a través de la lectura}

En la búsqueda de maneras para mejorar la capacidad para aprender a través de la lectura, los psicólogos cognoscitivos han incursionado dentro de las implicaciones más profundas de este proceso. Así han logrado determinar que el mismo puede ser afectado por muy diversos factores (Bransford, 1979 y Dansereau, 1980). Tomando como base estos aportes, así como las implicaciones prácticas que éstos pudieran tener para el diseño de una tecnología de instrucción, se podría decir que los factores que condicionan el aprendizaje de la información escrita se derivan de cuatro fuentes principales: los materiales de aprendizaje, el aprendiz, el ambiente y el propósito del aprendizaje.

\section{Dependientes de los materiales de aprendizaje}

Estos comprenden los factores que de alguna manera determinan la dificultad del lector para obtener la información contenida en los materiales escritos; es decir, los factores que están relacionados con su comprensibilidad. Estos incluyen, a su vez, factores relacionados con el contenido, tales como el tipo de disciplina a la cual pertenece, grado de abstracción, extensión, complejidad conceptual y carga inferencial; factores relacionados con la presentación del contenido, tales como el vocabulario empleado, la manera como están expresadas y relacionadas las ideas y conceptos, la forma de argumentar, la coherencia y relevancia de las proposiciones formuladas, el uso de la sintaxis, el estilo y las estructuras conceptuales presentes en el texto y el uso de los recursos de la comunicación (ejemplos, 
analogías, organizadores, signos de puntuación, etc.) y los factores relacionados con los aspectos tecnicográficos, tales como el formato, la diagramación, el tipo de letra, ilustraciones, tablas y gráficos.

\section{Dependientes del aprendiz}

En este grupo se incluye todo lo que se requiere para comprender la lectura y se derive de la naturaleza del propio lector. Tales serían, por ejemplo, su capacidad para procesar la información contenida en los textos escritos, su interés y motivación por la lectura en general y por leer y procesar el texto específico objeto del aprendizaje, su memoria y concentración en la lectura, su estilo cognoscitivo, sus conocimientos previos sobre el contenido del texto, sus condiciones físicas y fisiológicas relacionadas con la lectura, la saciedad y el control de cualquier necesidad física, fisiológica o psicológica que pudiera interferir significativamente en el aprendizaje, etc.

\section{Dependientes del ambiente}

Son todos aquellos factores derivados del ambiente que pueden afectar positiva o negativamente el aprendizaje. Incluyen las condiciones de temperatura, iluminación, ventilación, mobiliario y confort en general, al igual que la presencia de interferencias de cualquier tipo como ruidos, el timbre de la puerta, el teléfono, etc.

\section{Dependientes del propósito del aprendizaje}

Estos factores incluyen los relacionados con el motivo que lleva a la persona a involucrarse en el aprendizaje del contenido de un texto, tales como el origen del propósito (impuesto o autogenerado), su alcance (nivel de exigencia en cuanto a cantidad y profundidad en la comprensión), su precisión (claridad de las metas propuestas por el lector o de las instrucciones suministradas a éste), el tipo de propósito (para responder a una prueba escrita de selección múltiple o de desarrollo, para hacer una presentación oral en clase, para escribir un informe sobre una investigación), etc. 


\section{¿Cómo mejorar la capacidad para aprender el contenido de textos escritos?}

Múltiples investigaciones han logtado determinar que la eficiencia en el aprendizaje de textos escritos depende de la calidad del procesamiento a que se someta la información que éstos intentan transmitir. Este procesamiento depende, a su vez, de la eficacia de las estrategias cognoscitivas que utilice el aprendiz para realizar el procesamiento y de la habilidad con que éste aplique esas estrategias (Brown, 1982; Brown, Campione y Day, 1981, Morles et al., $1989^{\circ}$ y Morles, 1986b). Por lo tanto, para mejorar la capacidad para aprender a través de la lectura se hace necesario establecer, primero, cuáles son las estrategias cuyo uso se requiere ejercitar para lograr el mejoramiento y, segundo, cómo se debe conducir la ejercitación.

\section{¿Qué aspectos atender para lograt el mejoramiento de la capacidad para aprender?}

Como fuera discutido inicialmente, la capacidad para aprender mediante la lectura se basa en la habilidad del aprendiz para utilizar apropiadamente las estrategias de procesamiento necesarias para lograr la comprensión de la información, la retención o almacenamiento de esa información y la evocación de la misma, cuando las circunstancias lo requieran. Por esta razón, el mejoramiento de esa capacidad deberá incluir la ejercitación en el uso de estrategias apropiadas para procesar la información asegurando el aprendizaje, así como de estrategias para regular el desarrollo de ese procesamiento.

Estudios realizados permiten asegurar que tal ejercitación puede llegar a producir resultados significativamente positivos (Brown, 1982, Brown, Campione y Day, 1981). Por lo tanto, en el presente trabajo se propone que el mejoramiento de la capacidad para aprender el contenido de materiales escritos sea orientado por estas ideas.

Quien conduzca la ejercitación deberá tener presente que ésta será más efectiva si incluye el uso de estrategias de comprobada eficacia para 
asegurar el logro de cada uno de los componentes del aprendizaje de textos escritos; es decir, para la comprensión, la retención y la evocación. No obstante, conviene tener presente, igualmente, dos aspectos esenciales relacionados con este proceso: la inespecificidad de las estrategias y la especificidad de las características de cada aprendiz. Por inespecificidad de las estrategias se entiende que no existen estrategias exclusivas para cada componente del aprendizaje, aunque la manera como se conduzca la ejercitación podría asegurar la efectividad de alguna de ellas para determinado componente. Es decir, no existe una estrategia exclusiva para la comprensión, la retención o la evocación. Sin embargo, al conducir la ejercitación, una estrategia como, por ejemplo, relacionar la información contenida en el texto con conocimientos previos, pudiera utilizarse para comprender o para retener, según el énfasis que se dé a cada uno de estos componentes durante la ejercitación. Al tespecto conviene tener presente que si bien para comprender un texto lo importante es darle el mejor sentido a la información contenida en éste, para hacerla más significativa para retener y evocar esa información lo importante es establecer relaciones relevantes entre la nueva información y los conocimientos previos o esquemas conceptuales que posee el aprendiz.

Por especificidad de las características del aprendiz se debe entender que cada uno de éstos posee rasgos o circunstancias que le son propias y de allí lo específico de cada caso. Es decir, las características personales, los motivos, la manera como afectan al aprendiz las características de los materiales y del ambiente son únicas para cada estudiante. Por lo tanto, la manera de percibir, planificar, ejecutar y evaluar el aprendizaje será única para cada individuo, en cada circunstancia.

\section{(a) Estrategias para procesar la información}

Las estrategias para procesar la información son actividades mentales, no siempre conscientes, que realiza el lector para manipular y transformar la manera como está presentada la información en el texto escrito, con el propósito de hacerla más significativa y así lograr su comprensión, y la posterior incorporación de ésta a sus estructuras cognoscitivas. 
Mejoramiento de la Capacidad para Aprender

Es de suponer que la información aparece en el texto escrito en la forma como el escritor considera que es la más comprensible para los lectores a quienes va dirigida. Desafortunadamente esa forma puede no ser la más comprensible para muchos de ellos. Esto se debe a que, en primer lugar, la manera de suministrar la información responde a las características personales de quien escribe y, en segundo lugar, el modo de extraer el significado del texto es característico de la personalidad de quien lo lee. El grado de coincidencia que exista entre las características de ambos, el escritor y el lector, determinará en gran medida, la facilidad de procesar la información y lograr la comprensión del texto. Pero la coincidencia no se da frecuentemente y menos a lo largo de todo un texto; por lo que el lector, al no comprender, tiene dos posibilidades: intentar repetidamente obtener la información concentrándose en la búsqueda del significado del texto tal y como está escrito o actuar sobre el texto para transformar su lenguaje y/o la manera en que está presentado el contenido hasta lograr su comprensión.

Habrá quienes logren el significado de la información tal y como está escrito el texto, pero todo parece indicar que ellos constituyen sólo una minoría. La mayoría de los lectores manipula el texto escrito y transforma su lenguaje y/o su presentación realizando operaciones mentales para hacer que la información tenga más sentido para sí; es decir, para hacer que ésta sea más significativa para sí mismo y para obtener de este modo, su significado. Estas operaciones son conocidas como estrategias cognoscitivas para el procesamiento de la información. Estudios sobre la materia (O’Malley et al., 1984 y Morles et al, 1989) han permitido identificar cinco grupos de tales estrategias. Ellas son de "reorganización, elaboración, focalización, integración y verificación".

Cada grupo comprende una cantidad ilimitada de estrategias y aunque muchas de ellas son comunes a la mayoría de los lectores, existen otras que son de uso muy personal de cada lector y que responden, igualmente, a características muy personales de cada uno de ellos.

Conviene tener presente que no todas la estrategias que usa un lector son altamente eficientes. Algunas pueden no serlo y otras pueden ser 
eficaces sólo para ciertos lectores y en determinadas circunstancias. Por estas razones en este trabajo sólo se presentan algunas que han demostrado ser las más efectivas o que son usadas con mayor frecuencia por los buenos lectores. Estas son las que se considera que deberían ser incluidas en el entrenamiento, por juzgat que su empleo mejora significativamente la capacidad para comprender la lectura.

Sin embargo, hay que tener presente que el fin último del entrenamiento debe ser lograr que el lector no sólo use espontánea, oportunamente y con precisión las estrategias aquí mencionadas, sino que, además, genere y aplique exitosamente sus propias estrategias.

\section{Estrategias de reorganización}

Son las operaciones mentales que lleva a cabo el lector para dar a los componentes de la información (eventos, detalles, proposiciones, ideas, conceptos, etc.) un orden diferente porque aquél en que se encuentran no parece tener suficiente sentido lógico para él. Por esta razón, el lector tratará de reorganizarlos siguiendo un orden diferente que pudiera ser, según el caso, cronológico, espacial, jerárquico, inductivo, deductivo, causa-efecto, entre otros.

En vista de lo anterior, para ejercitar el uso de estrategias de reorganización el docente debería incluir el desarrollo de actividades que impliquen ordenat o reordenar eventos, ideas, detalles, conceptos, etc., de acuerdo con criterios variados, como tiempo, espacio, jerarquía, rango, número, orden alfabético, inductivo, deductivo, grado de abstracción, suficiencia, edad, o cualquiera otro que se adecúe a variados tipos de información. La ejercitación también podría incluir volver a escribir trozos o partes de ellos, dándole una estructura conceptual diferente (por ejemplo, inductiva frente a deductiva, descripción opuesta a narración) o simplemente, cambiar el orden de los componentes en alguna oración o frase. 


\section{Estrategias de elaboración}

Son las acciones mentales que ejecuta el lector con la intención de crear nuevos elementos que se relacionen con el contenido del texto para, de esa manera, hacer más significativo dicho contenido. Con el propósito de ejercitar al estudiante para que aprenda a usar este tipo de estrategias, el entrenamiento debería incluir actividades que lo adiestren para, por ejemplo, comentar para sí mismo el contenido de la lectura; el pensar en ejemplos sobre lo que está leyendo o en analogías que se asocien con ese contenido; visualizar el contenido de todo o de algunas partes del texto, formándose imágenes mentales sobre ello; evocar ideas o experiencias que conserva almacenadas en su memoria y que, igualmente, se relacionan con lo que está leyendo; agrupar o clasificar, asignando categorías a elementos de la información contenidos en el texto; transformar el lenguaje (parafrasear) de una o varias oraciones o de todo el texto para presentarlo en una manera diferente, utilizando, si fuese necesario, un lenguaje y una estructura conceptual distinta; formularse preguntas, hipótesis y predicciones; responder a preguntas; generar inferencias y conclusiones; pensar en implicaciones teóricas o prácticas de la información y otros.

\section{Estrategias de focalización}

Son aquellas estrategias que emplea el lector para precisar el significado de la información contenida en el texto escrito. Cuando la manera como está escrito un texto dificulta en alguna medida su comprensión, ya sea porque se presta a ambigüiedad o porque ésta implica el empleo de una cantidad mayor de palabras, oraciones o proposiciones, más ejemplos o más material redundante de la que el lector habría requerido, éste se vale de operaciones mentales que le permitan precisar su significado. Para lograr este fin, el lector procede a utilizar algunas estrategias mentales generales. Por ejemplo: considerando el propósito de la lectura, dará menos importancia a las partes del texto que juzgue redundantes o secundarias, o a las que constituyan ejemplos, y concentrará su atención en los aspectos que sean relevantes para el propósito que persigue. De 
esa manera extraerá la esencia de la información o el aspecto del contenido hacia el cual ha dirigido su atención al leer.

Por lo tanto, para ejercitar al estudiante en el uso de estrategias de focalización, el docente incluirá el desarrollo de actividades que contemplen la ubicación o reconstrucción de la idea central del texto o de las ideas principales y secundarias; leer precisando determinados aspectos (intencionalidad o modo del escritor) o buscando información específica para responder a ciertas preguntas o para conformar alguna hipótesis o predicción.

\section{Estrategias de integración}

Mediante el empleo de estas estrategias el lector busca unir en un todo coherente las partes de la información que obtiene a medida que va leyendo, a la vez que busca incorporarlas a los esquemas de conocimiento que él posee y que se relacionen con el tema objeto de la lectura.

Un ejemplo de tales estrategias lo constituye el agrupar en una sola las interpretaciones parciales que hace el estudiante mientras va leyendo. Otro sería el incorporar a los esquemas de conocimiento que el lector posee la información obtenida durante la lectura. Esto lo hace el buen lector tanto a medida que lee el trozo como al término de su lectura. El docente para ejercitar al estudiante en el uso de estrategias de integración debería proponerle actividades, tales como tomar nota de las interpretaciones parciales logradas mientras se lee y, al final de la lectura, integrarlas y formar una sola interpretación. Otra actividad podría consistir en dar una visión global sobre el contenido del texto que lee, integrándolo a los conocimientos que él ya posee sobre ese tema.

\section{Estrategias de verificacion}

Mediante las estrategias de verificación, el lector busca determinar hasta dónde las interpretaciones parciales hechas a lo largo de la lectura son coherentes entre sí y coherentes con el sentido común, la lógica, las opi- 
niones autorizadas y los esquemas de conocimiento que posea el lector y que se relacionen con el tema objeto de la lectura. Hasta dónde eventuales incongruencias entre las interpretaciones son debidas a errores suyos (del lector) o a detalles del propio texto. Al mismo tiempo, busca precisar si las interpretaciones parciales, lo mismo que la global, han considerado todos los elementos del contenido del texto involucrados en las interpretaciones.

Para entrenar al estudiante en el uso de estrategias de verificación el docente puede asignarle tareas en las cuales se le pida cotejar, tanto a medida que lee como al término de la lectura, la consistencia interna del texto; es decir, la armonía o grado de acuerdo entre las diversas interpretaciones parciales logradas y entre éstas la interpretación global del texto; cotejar, igualmente, la consistencia externa del texto; es decir, la coherencia de esas interpretaciones con la lógica, los esquemas de conocimiento del propio lector y las opiniones autorizadas que son del conocimiento de éste.

\section{(b) Estrategias para resolver problemas de procesamiento de la información}

Es de hacer notar que si bien se ha demostrado que el uso de las estrategias de procesamiento mencionadas facilita el aprendizaje de textos escritos, hay oportunidades en que, a pesar de su uso, el lector puede encontrar problemas para procesar algunas partes de un texto. Según Collins y Smith (1980) estos problemas se pueden referir a cuatro fuentes: comprensión de vocabulario, comprensión de oraciones, comprensión de relaciones entre oraciones y comprensión global del texto. A la vez, Maria y Mac Ginitie (1982) consideran que los niños presentan tres tipos de problemas:

- Dificultad para establecer relaciones apropiadas entre las oraciones o unidades semánticas de un texto.

- Rigidez en la confirmación de hipótesis o en la aplicación de esquemas del texto, formulados al inicio de la lectura.

- Rigidez en el uso de los conocimientos previos relacionados con el texto. 
Por otra parte, Morles, Amat, Donís y Urquhart (en prensa) encontraron que los problemas de procesamiento más frecuentes entre lectores adultos eran:

- Dificultad para encontrar el significado de palabras desconocidas.

- Dificultad para lograr la interpretación de alguna oración.

- Dificultad para establecer conexión entre oraciones.

- Incongruencia entre las interpretaciones de varias oraciones.

- Encuentro con palabras sin sentido en el texto.

Los problemas que puede llegar a confrontar una persona para procesar la información contenida en un texto escrito son muy numerosos y variados. Igualmente, las estrategias para resolver tales problemas pueden llegar a ser muy numerosas y variadas. Sin embargo, en el presente trabajo se sugiere que, para actuar más adecuadamente, se utilicen dos tipos de estrategias: unas generales y otras específicas.

Las generales incluyen estrategias que pudieran ser comunes para resolver eficazmente variados tipos de problemas. Tal sería el caso de releer todo el texto o la parte en la cual se ubica el problema hasta lograr su resolución, continuar leyendo en busca de más información que permita dar una solución al problema encontrado, parafrasear la parte del texto que presenta el problema (repetir esa parte del texto usando palabras, frases $\mathrm{u}$ oraciones diferentes), generar imágenes mentales relacionadas con la parte del texto que presenta el problema, formular bipótesis sobre lo que se supone significa esa parte del texto y continuar leyendo tratando de confirmar la certeza de la hipótesis formulada, pensar en analogias (en situaciones equivalentes a la expresada en el texto, pero de más fácil interpretación: por ejemplo, si el texto describe el aparato circulatorio, pensar en el acueducto de una ciudad), etc.

Las especificas incluyen las estrategias que pueden ser utilizadas para resolver problemas específicos de comprensión. Entre ellas se podrían sugerir las siguientes: 
Para establecer el significado de palabras desconocidas el docente podría ejercitar a los estudiantes para que encuentren su significado siguiendo cualquiera de las maneras siguientes: una, utilizar el contexto, es decir, hacer que ellos infieran el significado a partir de la información contenida antes o después de la palabra-problema. Dos, hacer que los estudiantes deduzcan su significado a partir de la estructura morfémica de la palabra. Tres, formular hipótesis sobre el significado de la palabra y seguir leyendo para ver si el significado supuesto es congruente con lo expresado posteriormente en el texto.

Para precisar las ideas principales de un texto el docente podría ejercitar a los estudiantes para que la ubiquen, descartando para ello, los detalles, la información redundante, la secundaria y los ejemplos, cuando cualquiera de éstos se encuentre presente.

Para encontrar una interpretación apropiada a una oración se ejercitará a los estudiantes para que relean la oración o partes más amplias del texto, generen imágenes mentales sobre lo expresado en la oración y parafraseen las oraciones, buscando darle sentido a éstas, y analicen las interpretaciones dadas a las oraciones próximas a las que constituyen el problema, tratando de darle una interpretación apropiada a éstas.

Para identificar los antecedentes de palabras o frases tales como cuyo, suyo, alli, aquél, así, de esa manera, por tal motivo, ejercitar a los estudiantes para que utilicen procedimientos como el siguiente:

Primero: Formularse una pregunta relacionada con la parte del texto en donde está ubicada la palabra o frase-problema, anticipando que su respuesta sea equivalente a esta palabra o frase.

Segundo: Buscar la respuesta a esa pregunta sabiendo que ésta debe concordar en número y género con la palabra o frase-problema y que, además, esta respuesta debe ser congruente con el resto del texto.

Los problemas presentados aquí son sólo algunos de los que podrían ser encarados por los estudiantes. De igual manera, las estrategias suge- 
ridas aquí son sólo las más frecuentemente utilizadas y las que han permitido obtener mejores resultados en algunos estudios conducidos. Sin embargo, obviamente, como no se han presentado todos los problemas ni todas las estrategias, sino una pequeña muestra de ellos, el docente debería partir de lo que aqui se ha planteado para identificar nuevos problemas y para ensayar la ejercitación de nuevas estrategias.

\section{(c) Estrategias para tegulat el proceso de aprendizaje de textos escritos}

El estado de alerta o de conciencia que manifiesta un estudiante sobre su proceso de aprendizaje y la regulación que ejerce sobre este proceso constituyen lo que es conocido como metacognición.

Tal estado implica el uso consciente e intencional de sus conocimientos y de sus habilidades para autorregular el desarrollo de su aprendizaje, con el objeto de asegurar su efectividad.

Obviamente, la metacognición tiene mayores posibilidades de producirse si el lector posee conocimientos adecuados sobre la naturaleza del proceso de comprensión y sobre la influencia de los factores que afectan su ejecución, así como sobre las estrategias de procesamiento, de resolución de problemas de procesamiento y de autorregulación de ese procesamiento. Sin embargo, una actitud positiva hacia ese procesamiento es condición indispensable para sus ejecución efectiva (Rothkopf, 1976). De otra manera, el procesamiento no se realizaría en toda su extensión.

La metacognición del estudiante puede ser incrementada mediante la ejercitación (Brown y Palincsar, 1982 y O’Malley et al, 1984). No obstante, para hacer más efectiva la ejercitación ésta debería incluir el suministro de:

- Conocimientos suficientes sobre, la naturaleza del proceso, sobre los factores que condicionan su ejecución y las estrategias para procesar la información, para resolver problemas de procesamiento 
y para regular ese procesamiento al intentar aprender el contenido de un texto escrito.

- Ejercitación en la aplicación de estrategias para ejecutar y regular el proceso de aprendizaje, lo cual incluye ejercitar al estudiante para que esté en capacidad de actuar espontánea, oportuna y eficazmente al seleccionar las estrategias ya sean éstas para procesar o para ajustar dicho proceso.

Los conocimientos y la ejercitación que se suministren al lector deberían permitirle estar enterado y alerta sobre las implicaciones de cualquier tipo que pudiera tener el procesamiento de la información y sobre cómo éstas influyen en la selección y aplicación de las estrategias.

Las estrategias en cuyo uso se ejercite al estudiante deberían referirse a tres fases del proceso de aprendizaje: Su planificación, su ejecución y su evaluación. Por tal razón, al concebir y conducir la ejercitación, el docente deberá incluir el desarrollo de actividades en las cuales el estudiante planifique, ejecute y evalúe conscientemente la aplicación de estrategias cognoscitivas para procesar la información.

\section{Ejercicios de Planificacion}

Estos deberían incluir la realización de actividades en las cuales el estudiante requiera precisar los alcances de lo que aspira lograr mediante la lectura (su propósito, objetivos y metas), estimar lo positivo o negativo de las condiciones del ambiente donde va a realizar la lectura (influencia de los factores provenientes del contexto o ambiente), analizar el material de aprendizaje para determinar sus características y las posibles exigencias de ese material para el logro del propósito, los objetivos y las metas y, finalmente, decidir sobre la manera como actuar luego de considerar los alcances de la tarea, las condiciones del ambiente, las características del material y sus propias características (capacidades intelectuales y físicas, dominio del contenido del texto: "cuánto sabe", "lo que no sabe", "lo que quiere o necesita saber sobre el tema", etc.). Como consecuencia de esa consideración el estudiante decidirá qué estrategias cognoscitivas utilizar 
y qué actividades ejecutar para procesar la información contenida en los materiales y para contrarrestar la acción de factores que pudieran interferir en la efectividad del aprendizaje.

\section{Ejercicios de ejecución}

Estos ejercicios estarán dirigidos al desarrollo de habilidades para realizar actividades mentales de tres clases: la autosupervisión del uso de las estrategias, tanto para procesar como para controlar la acción de factores condicionantes del aprendizaje, la autocomprobación de la efectividad del uso de esas estrategias y el autoajuste en el uso de esas estrategias (si alguna no fuese suficientemente efectiva, ajustar su aplicación y reemplazarla).

Para la conducción de la ejercitación el docente buscará que el estudiante realice actividades en las cuales estén implícitas la autosupervisión, la autocomprobación y el autoajuste, relacionadas con el uso de las estrategias durante la autorregulación del aprendizaje. Podría proponer al estudiante que exprese de alguna manera, durante la lectura, si está comprendiendo lo que lee, cuándo comenzó a comprender o cuándo dejó de comprender, si ha encontrado algún problema de comprensión, si las estrategias empleadas para procesar la información o para resolver problemas han sido eficaces para ir logrando el propósito de la lectura, si las interpretaciones parciales son congruentes entre sí. Es decir, hacer que el estudiante se haga consciente de su proceso de lectura.

Al mismo tiempo, le propondrá actividades en las cuales el estudiante reajuste la manera como ha estado desarrollando el proceso o que el estudiante reemplace alguna estrategia que había estado empleando, una vez que se modifiquen ciertas características de la tarea, del ambiente o de los materiales.

\section{Ejercicios de evaluación}

Entre los ejercicios de evaluación de aprendizaje el docente incluirá actividades en las cuales el estudiante evalúe el logro del propósito, de 
los objetivos o de las metas propuestas antes de iniciar la lectura, o que motivaron el desarrollo de ésta. Esto incluye determinar cuánto comprendió del contenido de la lectura realizada, hasta dónde logró el propósito, los objetivos y las metas (si es que ellos estaban establecidos y precisados) y cuánto recuerda el material que leyó.

Es importante que el docente y el propio estudiante estén conscientes de que si bien el desarrollo de las actividades propuestas hasta aquí pudiera implicar la realización de acciones no totalmente cognoscitivas como, por ejemplo, subrayar o escribir un resumen, ello se hace de esa manera para poder contar con elementos que permitan la supervisión y evaluación de las actividades llevadas a cabo.

Finalmente, conviene tener presente que de nada valdría suministrar al estudiante un amplio repertorio de estrategias para el logro de un aprendizaje efectivo, si él no está interesado o dispuesto a realizar el procesamiento necesario para aprender el contenido de un determinado texto escrito. De allí la importancia de atender al aspecto afectivo o motivacional del estudiante. En este sentido es necesario hacer consciente al estudiante de la importancia de mantener esa disposición durante todo el procesamiento y que esa disposición debe ser total y orientada hacia el logro del propósito que debe animar su aprendizaje. Esta disposición determinará en buena medida la calidad de los resultados del aprendizaje.

Al mismo tiempo es necesario, además de desarrollar en el estudiante una actitud favorable hacia el aprendizaje, asegurarse de que él esté en condiciones de contrarrestar los efectos de factores que pudieran afectar la calidad de su aprendizaje. Como fuera expresado inicialmente, estos factores pudieran estar vinculados a los materiales de aprendizaje, al ambiente o al propósito para el cual se intenta aprender. El estudiante requiere ejecutar acciones concretas para eliminar o reducir sus efectos $\mathrm{y}$ el docente debe suministrarle maneras apropiadas para asegurar la efectividad de esas acciones. 


\section{¿Cómo conducir la ejercitación para lograr el mejoramiento de la capacidad para aprender?}

El mejoramiento de la capacidad para aprender el contenido de textos escritos mediante la ejercitación lleva implícitos variados problemas que ameritan ser considerados a fin de garantizar la mayor efectividad de esos esfuerzos. Mediante esa consideración se buscará determinar, por una parte, cual será la modalidad de entrenamiento más apropiada para asegurar la mayor efectividad de la ejercitación y, por la otra, cuál será la ubicación más adecuada del programa.

\section{(a) Modalidades de entrenamiento}

El entrenamiento se puede conducir a través de variadas formas, las cuales se agrupan en cuatro modalidades principales: ejercitación, modelaje, instrucción directa o explicita e instrucción con autocontrol del aprendizaje. No obstante, conviene aclarar que sería prácticamente imposible el uso de modalidades puras. Por lo general el instructor combina estas modalidades de acuerdo con su criterio, aunque, al conducir el entrenamiento, imponga el predominio de algunas de ellas.

La ejercitación se caracteriza por enseñar al estudiante el uso de determinadas estrategias mediante la ejecución de tareas en forma oral o escrita. Para resolver una tarea asignada, el estudiante debe emplear la estrategia propuesta, pero no se le dan mayores explicaciones y no hay un instructor que le muestre la manera de utilizar la estrategia. El instructor sólo asigna la tarea, vigila su cumplimiento y evalúa los productos del trabajo realizado. Esta modalidad es muy usada por los docentes en la instrucción ordinaria, especialmente en los niveles inferiores de la educación.

El modelaje es la forma de enseñanza en la cual el instructor u otra persona "modela", es decir, "actúa" ante los estudiantes para mostrar la manera como se utiliza una estrategia determinada. En esta modalidad se resta importancia a la fundamentación y justificación de la estrategia a enseñar y se insiste en que el estudiante "copie" la forma como actúa el 
"modelo". Un ejemplo de modelaje sería el caso del docente que pretende enseñar el uso de las autopreguntas, como estrategia para comprender la lectura, y sigue la manera que plantea Flavell (1979), de hacer que los niños "piensen en voz alta". En este caso, el docente se formulará preguntas (en voz alta) a medida que lee un cuento. Por ejemplo dirá: "Ay, ¿qué irá a pasar ahora?", "¿por qué hizo esto?” y buscará dar respuesta a estas preguntas, a medida que lee el cuento.

Dansereau (1980) señala tres formas de modelaje: una que consiste en la presentación por parte del instructor, del producto final de un ejercicio, el cual ha sido realizado utilizando correctamente determinada estrategia. El producto va acompañado de anotaciones que orientan sobre cómo fue logrado éste. Otra forma sería el "copiar" la manera como actúa un experto al aplicar una estrategia específica. Un ejemplo de esta forma sería la lectura del cuento comentada anteriormente. Una tercera forma sería la que Dansereau denomina "modelaje interactivo". Este caso se presenta cuando un número de estudiantes trabaja en grupo para aplicar determinada estrategia y, mientras realizan el trabajo, éstos se proveen retroalimentación mutua.

En el modelaje, al igual que en la ejercitación, la importancia que se da a la información sobre cada estrategia y sobre el entrenamiento en sí es muy limitada. Estas modalidades podrían incluirse en lo que Brown y Palincsar (1982) denominan "entrenamiento ciego".

La instrucción directa o explícita consiste en informar al estudiante directamente sobre todo lo relacionado con el entrenamiento y la estrategia sobre cuyo uso se desea entrenar; es decir, sobre el plan de entrenamiento, su fundamentación, contenido y propósito; sobre las estrategias a emplear, su naturaleza, el por qué y cómo de su empleo y sobre los progresos logrados por el estudiante durante el desarrollo del programa.

En esta instrucción se provee al estudiante toda la información requerida. Ello significa que, al nivel de la información, no habrá ningún problema. Brown y Palincsar (1982) denominan a esta modalidad "entre- 
namiento informado" y la consideran como una metodología de alta efectividad.

La instrucción con autocontrol del aprendizaje es similar a la instrucción directa y explícita, pero incluye un componente fundamental. Este es el entrenamiento en el uso de la autosupervisión y la autorregulación, durante la aplicación de las estrategias que se enseñan. Además, incluye la autoevaluación de los resultados, continuamente y al final de la aplicación del programa. En esta modalidad, por lo tanto, se ejercita al estudiante en el empleo de estrategias cognoscitivas y metacognoscitivas, tales como las enumeradas en el presente trabajo. Los estudios han demostrado que la instrucción con autocontrol del aprendizaje es la modalidad más efectiva (Brown y Palincsar, 1982) por lo que el entrenamiento debe propiciar esta modalidad.

\section{(b) Ubicación del programa de mejoramiento}

Respecto a la ubicación del programa de entrenamiento, conviene señalar que existen por lo menos tres posibilidades. Una, tratarlo como una asignatura regular y aislada del currículum del grado respectivo; dos, incluirlo como una actividad ordinaria dentro del programa de lectura respectivo (cuando éste exista) y tres, incluirlo como actividad regular comprendida dentro de cada una de las asignaturas que forman el currículum del nivel educativo correspondiente. De las tres opciones la que pareciera más apropiada es la última. Incluirla como una actividad regular dentro de cada asignatura va a permitir la vinculación de las estrategias de procesamiento de la información con temas específicos. Esto sería más efectivo porque asegura la adecuación de las estrategias a diferentes tipos de materiales y temas. Además, se contaría con un campo real de aplicación para las estrategias.

Por otra parte, un programa así ubicado garantizaría la internalización y consolidación del uso de las estrategias, lo cual constituye una fase fundamental de entrenamiento. Por supuesto, tal ubicación requiere contar con personal, en todas las asignaturas, debidamente entrenado y motivado 
para la aplicación del programa, y contar con el tiempo necesario para esa aplicación.

En conclusión, es un hecho irrebatible que la educación depende cada día más de la capacidad de los estudiantes para aprender a través de la lectura. Es igualmente irrebatible afirmar que los estudiantes presentan marcadas deficiencias en su capacidad para aprender el contenido de los materiales escritos. Por estas razones surge como impostergable la necesidad de mejorar esta capacidad en la población estudiantil.

Una manera suficientemente comprobada de actuar para lograr ese mejoramiento lo constituye la ejercitación en el uso de estrategias mentales para procesar la información contenida en los textos escritos. Pero, a pesar de la efectividad de esas estrategias, es necesario ejercitar al estudiante, igualmente, en el uso de estrategias para planificar, ejecutar y evaluar el aprendizaje.

Un aspecto muy importante para asegurar la efectividad de la ejercitación lo constituye la ubicación de esta actividad dentro del trabajo del aula. Los estudios han demostrado que la mayor eficiencia de un programa de entrenamiento se logra cuando éste se ejecuta como una actividad rutinaria, dentro del desarrollo ordinario del plan curricular de cada asignatura. Por tal razón cada profesor debe ejercitar a los estudiantes en su propia asignatura para que usen las estrategias mentales que, en forma general, se especifican en este trabajo y para hacerlo, el profesor podrá seguir las ideas que aquí brevemente se exponen.

\section{Referencias}

Bransford, J. D. (1979). Human Cognition. Belmont, California: Wadsworth Publishing Company.

Brown, A. L. (1982). Learning how to learn from reading. En Langer, J. A. y Smith-Burke, M.T. (editores), Reader Meets Author-Bridging the Gap". Newark : International Reading Association. 
Brown, A., Campione, J. y Day. (1981). Learning to learn: On training students to learn from texts. Educational Rescarcher, 10, febrero.

Brown, A. L. y Palincsar, A. S. (1982). Inducing strategic learning from texts by means of informed self-control training. Topics in Learning and Learning Disabilities, 2, p.p.1-27.

Collins, A. y Smith, E. E. (1980). Teaching the process of reading comprehension. En B.B. N. Inc. (Eds.). Project Intelligence; The development of procedures. Boston: Harvard University Press.

Dansereau, D. (1980). Learning strategy research: Trabajo presentado en la NIE-LERDOC Conference on Thinking and Learning Skills. Universidad de Pittsburgh.

Flavell, J. (1979). Metacognition and cognitive monitoring: A new area cognitive development inquiry. American Psychologist, 34, (10).

Maria, K. y Mac Ginitie, W. H. (1982). Reading comprehension Disabilities: Knowledge structures and non-accomodating text processing strategies. Annals of Dyslexia, 32.

Morles, A. (1991). El desarrollo de habilidades para comprender la lectura y la acción del docente. En A. Puente (editor). Comprensión de la Lectura y la Acción Docente. Madrid: Fundación Germán Sánchez Ruipérez Morles, A. (1986a). Entrenamiento en el uso de estrategias para comprender la lectura. Revista Latinoamericana de Lectura (Lectura y Vida). 7, (2).

Morles, A. (1986b). El desarrollo de la capacidad para comprender la lectura. Revista de Tecnologia Educativa, IX, (4).

Morles, A., Amat, M., Donís, Y. y Urguhard, R. (en prensa). Resolución de problemas de procesamiento de la información durante la lectura. Revista Latinoamericana de Lectura (Lectura y Vida).

Morles, A., Amat, M., Donís, Y. y Urquhart, R. (1989). Estrategias para la comprensión del texto escrito. Revista Investigación y Postgrado, 4, (1). Enero.

O’Malley, J. M. y Chamot, A., Stewner, S., Russo, R. y Kupper, L. 1984). A study of learning strategies with of English as a second language. Trabajo presentado en la Conferencia Anual de la AERA. Nueva Orleans, L. A 
Mejoramiento de la Capacidad para Aprender

Rothkopf, E. Z. (1976). Writing to teach and reading the learn: A perspective on the psychology of written instruction. En The Psychology of Teaching Metbods The seventy fifth yearbook of the National Society for the Study of Education (NSSE). Chicago. 